\title{
Como avaliar a deliberação online? Um mapeamento de critérios relevantes
}

\author{
Rafael Cardoso Sampaio \\ Doutorando em Comunicação e Cultura Contemporânea \\ Universidade Federal da Bahia \\ Samuel Anderson Rocha Barros \\ Mestrando em Comunicação e Cultura Contemporânea \\ Universidade Federal da Bahia \\ Ricardo Morais \\ Doutorando em Ciências da Comunicação \\ Universidade da Beira Interior - Portugal
}

\begin{abstract}
Resumo: Este artigo explora os critérios utilizados nas pesquisas da área de Deliberação Online. Através de uma ampla revisão de literatura, foram selecionados os 59 artigos que elencam os indicadores a serem medidos em discussões na internet. Nestes artigos, foram encontrados, ao todo, 369 critérios, com média de 6,25 por artigo e desvio padrão de 2,69. Em um segundo momento, o esforço da pesquisa se deu no agrupamento de tais critérios em categorias mais amplas e conectadas aos princípios deliberativos. Os critérios foram então resumidos a 8 categorias, a saber: Justificação, Reciprocidade, Reflexividade, Respeito, Pluralidade, Igualdade, Informação e Tópico. Conclui-se que, apesar dos muitos critérios utilizados, não se trata de uma dispersão dos estudos de deliberação online. Este resultado está ligado às diferentes correntes teóricas da democracia deliberativa, à difícil operacionalização dos critérios, à necessidade de critérios específicos para os diferentes contextos e objetos de análise e, em vários casos, à simples diferença de taxonomia.
\end{abstract}

Palavras-chave: democracia deliberativa; deliberação online; comunicação política; esfera pública

\begin{abstract}
This paper explores the main criteria used in researches in the field of online deliberation. Through an extensive literature review, we selected 59 articles that list indicators to assess discussions in the internet. In these articles were found, altogether, 369 criteria, with an average of 6,25 per item and standard deviation of 2,69. In a second stage, the research effort is made to group these criteria into broad categories and connected to the deliberative principles. The criteria were summarized in eight categories, namely: Justification, Reciprocity, Reflexivity, Respect, Pluralism, Equality, Information and Topic. We conclude that despite the many criteria used, one cannot say there is an excessive dispersion at online deliberation studies. This result is linked to different deliberative democracy approaches, the difficult to operationalize the criterion, the need for specific criterion for different contexts and objects and, in many cases, the mere difference of taxonomy.
\end{abstract}

Keywords: deliberative democracy; online deliberation; political communication; public sphere

OPINIÃO PÚBLICA, Campinas, vol. 18, n², novembro, 2012, p. $470-489$ 
SAMPAIO, R. C.; BARROS, S. A. R.; MORAIS, R. Como avaliar a deliberação online?...

\section{Introdução ${ }^{1}$}

As teorias democráticas contemporâneas têm procurado alternativas para tornar a democracia mais participativa, aproximando os eleitores do governo e das decisões. A corrente deliberacionista tem surgido como uma alternativa, que confere aos cidadãos a possibilidade de deliberarem racionalmente sobre as decisões que thes dizem respeito (HABERMAS, 1997).

O desenvolvimento das tecnologias digitais de comunicação tem reforçado o debate sobre a participação civil nas democracias contemporâneas. Estes novos meios possuiriam maiores potencialidades de interação, que teriam "implicações potenciais para a motivação, habilidade e oportunidade de os cidadãos se tornarem envolvidos na vida pública" (DELLI CARPINI, 2000, p. 346-347).

No entanto, grande parte destes novos estudos centrados na análise das possibilidades da online deliberation (OD) apresenta como base teórica os requisitos antes estabelecidos para a definição da esfera pública, os quais procuram adaptar ao ambiente online da internet e às suas especificidades. Estes requisitos, indicados por Habermas e sistematizados por diversos autores, vão orientar as diferentes iniciativas que procuram avaliar a deliberação nos ambientes online.

Assim, o percurso deste artigo dá-se a partir de uma breve apresentação e contextualização da corrente teórica deliberacionista, apresentando os seus principais pressupostos, para, em seguida, tratar das potencialidades dialógicas dos meios digitais e as possibilidades da OD. Posteriormente, são apresentados a metodologia e o corpus, assim como os resultados do levantamento e do agrupamento dos diferentes critérios em oito categorias principais.

\section{Democracia Deliberativa e a Deliberação Online}

No início da década de 1980, Bessette (1980) cunhou pela primeira vez a expressão "democracia deliberativa" e surgiram os primeiros trabalhos (MANIN, 1987; COHEN, 1989) utilizando esta expressão de acordo com as diferentes interpretações da Constituição Americana. No entanto, apenas na década seguinte, esta corrente ganha um lugar de destaque na teoria política democrática (SILVA, 2004). De um modo geral, a democracia deliberativa distingue-se das outras teorias democráticas pelos pressupostos de sua base:

\footnotetext{
"Em primeiro lugar, a sua insistência na noção de "debate racional" enquanto procedimento político por excelência, em vez da noção de "compromisso entre interesses divergentes". Em segundo lugar, como sublinha John Elster, ao invés dum acto privado como é o voto, a democracia deliberativa aposta num acto público enquanto acto político por excelência: a troca livre e pública de argumentos" (SILVA, 2004, p. 2).
}

Por sua vez, os textos do filósofo alemão Jürgen Habermas sobre o modelo deliberativo se tornam uma grande referência mundial, influenciando grandes discussões na filosofia e ciência política. Habermas indica a relevância de um modelo de democracia que, de um lado, não abdique da interação

\footnotetext{
${ }^{1}$ Uma versão anterior deste artigo foi apresentada no GT "Ciberpolítica, ciberativismo e cibercultura" da Anpocs em 2011. Os autores agradecem às sugestões e revisões de Jamil Marques, Marcus Abílio, Ricardo Fabrino e dos pareceristas anônimos da Opinião Pública.
} 
OPINIÃO PÚBLICA, Campinas, vol. 18, n², novembro, 2012, p. $470-489$

forte e discursiva entre cidadãos e representantes para a formação da opinião e que, de outro, não deixe de reconhecer direitos, liberdades e reivindicações individuais (HABERMAS, 1997). É neste contexto que a democracia de cunho deliberativo ressalta a ideia fundamental de esfera pública, cuja ênfase se encontra na necessidade de participação da esfera civil na produção da decisão política.

O processo de deliberação representa um aprofundamento da noção de esfera pública, procurando, através do debate racional e da troca pública e livre de argumentos, encontrar um caminho para legitimar os processos de produção da decisão política nas democracias (BoHMAN, 1996). Este processo de deliberação, segundo Habermas, deve estar de acordo com uma "ética do discurso", isto é, com um conjunto de princípios que se resumem nos seguintes aspectos: todos os participantes devem ser capazes de formular razões próprias e passíveis de serem compreendidas e aceitas; de iniciar debates e interpretar as suas necessidades de maneira reflexiva, expondo os seus interesses sob uma perspectiva generalizante, sem que haja desigualdades de acesso e de poder (HABERMAS, 1997).

Os procedimentos deliberativos, para Habermas, são a forma de gerar integração social, pois esta não pode ser gerada apenas pela ação comunicativa, apenas pelo desejo dos cidadãos de se entenderem. Os procedimentos permitem que o poder gerado comunicativamente se transforme em poder passível de ser empregado em termos administrativos. Há, então, um procedimento ideal de dar e receber razões.

A deliberação é a busca pela "melhor solução", ou ainda, a mais válida, justa, verdadeira. Ela pode trazer à tona modos de lidar com conflitos que de outra forma não encontrariam solução. Os processos de formação da opinião e da deliberação influenciam as preferências dos participantes, pois podem selecionar os temas, as contribuições, as informações e os argumentos. Assim, idealmente, apenas os "válidos" conseguem atravessar os filtros de negociações e dos discursos racionais, ganhando importância para as tomadas de decisões (HABERMAS, 1997).

Neste contexto, as concepções deliberativas têm se destacado enquanto modelos nos quais a opinião pública legitima as decisões políticas, uma vez que procuram a melhoria da democracia pelo debate público, com o objetivo de uma justificação racional das decisões políticas. Esta ideia da deliberação se afasta do conceito de maioria, tão caro às teorias da representação, substituindo-o por um entendimento comum alcançado através da argumentação racional.

\footnotetext{
“De acordo com a maioria dos teóricos da democracia deliberativa, a decisão política é legitimada quando as políticas são produzidas num processo de discussão pública e debate entre cidadãos e os seus representantes, indo além do mero interesse pessoal, refletindo o interesse geral ou o bem comum" (BOHMAN, 1996, p.4, tradução nossa).
}

Neste sentido, o que os autores da corrente deliberativa (BOHMAN, 1996; GASTIL, 2000; HABERMAS, 1997) consideram um progresso, no modelo que defendem, em relação às concepções anteriores, é a existência de mecanismos que contemplem a participação dos cidadãos, que é importante para que se afirmem questões de legitimação democrática.

Buscando alternativas ao modelo de deliberação habermasiano, há uma linha de pensamento que afirma que a razão não pode ser o único elemento a compor a deliberação. Segundo Dryzek (2007), 
SAMPAIO, R. C.; BARROS, S. A. R.; MORAIS, R. Como avaliar a deliberação online?...

os modelos de deliberação baseados na excessiva racionalidade e justificação (RAwLS, 2000, 2002; GUTTMAN, THOMPSON, 2007) são muito exigentes, pois tendem a excluir a coerção, a decepção, a estratégia e a manipulação do processo deliberativo. De modo semelhante, buscamos aqui definições mais contemporâneas de deliberação democrática, que possam aceitar diversos tipos de comunicação, como a retórica, contar histórias ou mesmo o uso de boatos e piadas (DRYZEK, 2007). Conforme Dryzek, apenas é necessário que essas formas alternativas de deliberação sejam não coercitivas, capazes de induzir a reflexão e de conectar pontos de vistas e experiências particulares com princípios e questões mais gerais (DRYZEK, p. 241, 2007).

Mansbridge (2007) faz proposta semelhante ao explicar que as emoções se entrecruzam com a cognição, de modo que o discurso emotivo também pode fazer parte do discurso racional na deliberação. As emoções, segundo a autora, nos ajudam a pensar, a pesar a importância das alternativas, sentir empatia e decidir. Logo, o processo de dar razões deve ser plural na sua própria natureza, acolhendo também as emoções.

Mansbridge rejeita a ideia de um bem comum muito definido da maneira originalmente proposta por autores deliberacionistas tradicionais, como Cohen (1997). O bem comum, afirma, também pode ser plural, tendo inúmeras definições. A autora acredita que a busca desse bem comum é mais compatível com barganha e agregação que os conceitos racionais aceitam. Finalmente, a deliberação pode ser plural nos fins e nos meios. O objetivo da deliberação não é apenas construir ligações entre indivíduos ou apenas buscar o consenso racionalmente motivado, mas também iluminar os interesses em comum e aqueles em conflito.

De tal maneira, o processo deliberativo pode incluir a negociação e até a barganha, pois eles podem ajudar os participantes a entenderem melhor os pontos de vista dos outros e até seus próprios. $\mathrm{E}$ se a ideia de bem comum estiver ligada à melhor solução para o maior número de pessoas, a barganha e a negociação devem ser importantes partes da comunicação mútua para forjar e descobrir esse bem (MANSBRIDGE, 2007, p. 264).

Warren, por sua vez, também tenta ampliar as possibilidades para a realização da deliberação. O autor afirma que devemos esperar que os participantes entrem na comunicação com intenções estratégicas, pois isso define uma questão política na qual eles discordam e que há apostas que os motivam a tentar vencer. As instituições devem canalizar a intenção estratégica em falas, na esperança de que os participantes só possam alcançar seus caminhos através das falas, o que os levaria a tentar a persuasão. Em outras palavras, Warren sugere que nos afastemos da ideia de que a intenção original dos participantes é vital para o resultado da deliberação. Segundo sua concepção, mesmo se houver falas que não sejam deliberativas originalmente, elas podem ser capturadas para produzir dinâmicas que sejam deliberativas na função (WARREN, 2007, p. 278). Ou seja, a deliberação não precisa ser totalmente isenta da ação estratégica e unicamente voltada para o entendimento, como se concebia nas deliberações ideais (COHEN, 1997) e na ação comunicativa (HABERMAS, 1997).

Em resumo, esses autores defendem que outras formas de comunicação podem e devem ser incluídas no processo deliberativo. Além disso, eles tentam evidenciar que a deliberação pode servir a diversos fins, não podendo se afirmar que se trata de um único processo com um objetivo principal. 
OPINIÃO PÚBLICA, Campinas, vol. 18, n², novembro, 2012, p. $470-489$

Segundo Dryzek, a deliberação pode facilitar aos indivíduos trocarem suas preferências, aumentar a legitimidade democrática, aumentar a racionalidade para resolução de problemas, fazer a escolha coletiva mais maleável, aumentar a igualdade política, o aprendizado social e fomentar melhores cidadãos. É importante acrescentar que dificilmente uma deliberação poderá servir a todos esses objetivos, sendo importante definir a tarefa da deliberação (DRYZEK, 2007; WARREN, 2007).

Esta corrente ganha ainda maior respaldo quando as novas tecnologias, e em especial a internet, emergem como potencialmente capazes de fortalecerem valores democráticos. Assim, à internet tem sido atribuído um conjunto de inúmeras potencialidades, que vão desde a possibilidade de autoexpressão e estabelecimento da comunicação sem coerções, passando pela sua enorme capacidade interativa e de instantaneidade, até a memória e a capacidade de armazenamento de informação, aspectos que, reunidos, permitiriam, potencialmente, uma maior agilidade, intensidade, alargamento e aprofundamento da interação social (GOMES, 2008).

É neste contexto que surge a deliberação online, ou seja, numa época em que os cientistas sociais, explorando a possibilidade da democracia deliberativa, estão também interessados na relação entre tecnologia e democracia.

E tal como os teóricos da democracia deliberativa reconheciam que o conceito de deliberação tinha diversos significados (DRYZEK, 2007), também os estudiosos da OD prestam atenção às diferentes formas através das quais as novas tecnologias podem proporcionar a aplicação dos princípios deliberativos.

Assim, pode-se afirmar que o campo da deliberação online (OD) está dividido em cinco objetivos primários. Primeiramente, parte dos pesquisadores compara as deliberações online com as presenciais, procurando comprovar (por pesquisas quase-experimentais empíricas) que ambas podem incrementar os conhecimentos dos participantes sobre os assuntos, a eficácia política e a vontade para participar na política (GASTIL, 2000; DAHLBERG, 2004; BAEK et al, 2011).

O segundo grupo analisa a deliberação online natural (in the wild). Busca analisar a qualidade dos debates online que ocorrem espontaneamente entre cidadãos interessados e compreender as características que facilitaram ou dificultaram a deliberação qualificada entre os participantes (GRAHAM, 2008; JANSSEN, KIES, 2005; WILHELM, 2000).

O terceiro conjunto analisa a deliberação em programas participativo-deliberativos ou em fóruns online hospedados em sites institucionais. Nesses casos, geralmente, a apreensão da OD visa verificar se o processo participativo conseguiu abrigar uma deliberação qualificada, buscando-se compreender os motivos para tais resultados (DAHLBERG, 2004; JENSEN, 2003; WRIGHT, STREET, 2007).

Quarto, há um agrupamento de estudos que procura investigar como o design e a estrutura afetam a qualidade da deliberação e os seus resultados. Apreende-se às características estruturais que poderão ser controladas por criadores dos espaços de debate, tais como formulários (quadros de avisos contra as listas de e-mail), abertura (com/sem registro), diversidade (participantes homogêneos vs. heterogêneos), regulação (com/sem moderadores), e assim por diante (DECINDIO, PERABONI, 2010; JANSSEN, KIES, 2005; NOVECK, 2003). 
SAMPAIO, R. C.; BARROS, S. A. R.; MORAIS, R. Como avaliar a deliberação online?...

Há um quinto objetivo, que, segundo nossa avaliação, é o mais recente entre eles: trata-se da avaliação de mapas de argumentos criados por softwares². Ao invés da codificação manual (humana) dos fóruns, tais softwares são capazes de avaliar um número muito superior de mensagens e de gerar interligações entre os argumentos, ou ainda, mapas visuais que demonstram a utilização dos argumentos em determinada discussão (LoukIS et al, 2009; KARAMANOU et al, 2011; TAMBOURIS et al, 2011).

Existem, assim, várias formas de tratar a questão da OD e, perante esta variedade de caminhos de investigação, cabe salientar que este artigo não pretende ser exaustivo, mas objetiva fazer um levantamento dos trabalhos existentes e identificar os indicadores mais visitados na literatura.

\section{Que critérios para medir a deliberação online?}

Os instrumentos para medir a OD têm aumentado à medida que surgem novos trabalhos e experiências, e nesse sentido, consideramos que existem micro e macroanálises, assim como medidas de deliberação diretas e indiretas (BLACK et al, 2009). Assim, se as microanálises estudam a qualidade da deliberação analisando o conteúdo dos comentários dos participantes durante o processo deliberativo, as macroanálises solicitam colaboradores para fazerem avaliações da discussão como um todo (surveys).

Por outro lado, os estudos que utilizam medidas diretas focam o decorrer do processo de deliberação, enquanto os estudos que usam medidas indiretas avaliam a deliberação com base nos seus antecedentes ou resultados da discussão, por exemplo, medindo as mudanças nas preferências dos participantes após a deliberação (BACHTIGER et al, 2009).

As duas estratégias apresentam vantagens e desvantagens. Se as estratégias de medição indiretas e macroanalíticas têm a vantagem da rapidez no recolhimento dos dados, por outro lado, uma desvantagem simples tem a ver com o fato de o investigador apenas poder tirar conclusões observando os antecedentes ou os resultados de um processo deliberativo. Por sua vez, as estratégias microanalíticas têm como principal inconveniente a dificuldade na obtenção de confiabilidade da codificação, ou seja, procuram definir alguns critérios ideais e depois testar a qualidade da deliberação de acordo com esses critérios (BACHTIGER et al, 2009).

Grande parte das pesquisas apresenta indicadores analíticos baseados nos princípios normativos formulados por Habermas, embora parte delas utilize os princípios apenas como guia na elaboração de uma proposta metodológica, adicionando outros princípios àqueles anteriormente listados, mostrando que eles são úteis, porém limitados, para avaliar as comunicações online, pois ignoram as realidades desse tipo de espaço de conversação.

\footnotetext{
${ }^{2}$ Ver Benn, Macintosh (2011) para uma discussão sobre essa nova forma de avaliação da deliberação online e sobre os principais softwares utilizados.
} 
OPINIÃO PÚBLICA, Campinas, vol. 18, n², novembro, 2012, p. $470-489$

\section{Metodologia}

A pesquisa aqui relatada foi baseada na revisão de literatura, conforme revisão semelhante realizada por Trénel (2004). Entretanto, foi vital agregar um número representativo de artigos. Para tanto, optou-se pelo uso de instrumentos de pesquisa amplos, que verificassem diversas bases de conhecimento simultaneamente. Foram escolhidos o Portal de Periódico CAPES, o Google Acadêmico (Scholar) e o All Academic Research ${ }^{3}$. No primeiro, foram selecionadas as bases de "Ciências Sociais Aplicadas", "Ciências Humanas" e "Multidisciplinar"4, enquanto nos outros dois foram verificados todos os artigos com acesso. As palavras-chave iniciais foram: "Online+Deliberation" e "Deliberação+Online". Posteriormente, também foram pesquisadas: "e-Deliberation", "Electronic+Deliberation" e "Virtual+Deliberation".

A prospecção foi realizada em dois momentos distintos. Inicialmente, de outubro a novembro de 2010 e, depois, entre novembro e dezembro de $2011^{5}$. Ao fim dos levantamentos, foram encontradas 215 referências no Portal de Periódicos, 651 no Google Acadêmico e 16 no Al/ Academic (há referências cruzadas, repetidas). Durante a fase inicial, o resumo dos trabalhos foi usado como forma de seleção. $\mathrm{Na}$ dúvida (ou seja, nos casos em que não fosse possível a avaliação exclusivamente através do resumo), o pesquisador deveria verificar o texto em busca dos critérios. Dessa forma, foram selecionados 74 artigos iniciais. A segunda fase constou na leitura dessas pesquisas, verificando se condiziam com a proposta do artigo, o que já resultou na exclusão de alguns artigos do corpus final. Além disso, foram excluídos os artigos de um mesmo autor que faziam uso de critérios idênticos entre si. Por exemplo, os critérios por Sampaio (2010) foram reaplicados em Sampaio et al (2010). Nesse caso, apenas o segundo trabalho foi selecionado para a análise. Finalmente, na terceira fase, foi realizada uma segunda varredura pelos portais de busca mencionados e também pelas referências dos artigos, que ainda não compunham o corpus. Após tais etapas, 59 artigos foram selecionados para o corpus (Anexo 1), sendo exatamente 50 em língua inglesa e 50 escritos em português (todos por brasileiros) ${ }^{6}$.

Cada pesquisador ficou responsável por preencher uma ficha de análise sobre os artigos contendo: nome do artigo, autor, local de publicação (revista, evento etc.), local de aplicação da pesquisa, critérios utilizados para o estudo e resultados obtidos em porcentagem. Este artigo apenas com os critérios. Ademais, foram verificados apenas critérios inerentes à análise dos discursos realizados em meio digital. Indicadores ligados à estrutura do fórum, como identificação, moderação, agendamento, liberdade de expressão e afins não foram contabilizados, por serem considerados fatores externos da deliberação (JANSSEN, KIES, 2005), não estando, portanto, no escopo da pesquisa aqui relatada.

\footnotetext{
${ }^{3} \mathrm{O}$ Al/ Academic Research é um banco de dados onde se podem encontrar diferentes tipos de publicações acadêmicas como artigos de revistas, comunicações apresentadas em congressos e documentos de trabalho. O All Academic Research é um dos serviços disponibilizado pela $A / /$ Academic $/ n c$, uma prestadora de serviços de aplicações especializada em ferramentas para a comunidade de pesquisa acadêmica.

4 Através do Portal CAPES foram checadas as seguintes bases: Academic Search Premier. ASP (EBSCO), Cambridge Journals Online, Highwire Press, Oxford Journals (Oxford University Press), Scielo.org, Science (AAAS), ScienceDirect (Elsevier), Wiley Online Library, Emerald Fulltext (Emerald), JSTOR Arts \& Sciences, OECD - iLibrary, Project Muse, Education Full Text (Wilson), PsyArticles (APA) e Soc/NDEX with Full Text (EBSCO).

${ }^{5} \mathrm{O}$ segundo levantamento foi realizado durante a revisão sugerida por pesquisadores da Anpocs e pelos pareceristas da Opinião Pública.

${ }^{6}$ Alguns pesquisadores portugueses tratam do tema da deliberação online (ver Labcom, 2011), porém, não foram encontrados artigos em português com critérios de OD. A exceção foi o artigo de Ferreira (2011), mas este estava na língua inglesa.
} 
SAMPAIO, R. C.; BARROS, S. A. R.; MORAIS, R. Como avaliar a deliberação online?...

\section{Resultados}

Dos 59 trabalhos, 22 foram publicados em periódicos, 27 apresentados em eventos, 4 são capítulos de livros, 1 dissertação de mestrado e 2 teses de doutorado, além de 3 trabalhos classificados como relatórios de pesquisa. Entre os periódicos, o destaque foi o Javnost - The Public, com 5 artigos, e em seguida, o New Media \& Society com 3 artigos. Entre os eventos, destaque para Annual Conference on Online Deliberation com 7 artigos, seguido pelo encontro da International Communication Association com 4 artigos, Political Studies Association Conference com 3 artigos, e Internet Research com 2 artigos.

Como esperado, a maior parte das pesquisas $(\mathrm{N}=29)$ foi aplicada em fóruns assíncrono online (sendo 6 da Usenet), 5 em seções de comentários (Em jornais online ou plataformas específicas) e 4 em listas de e-mails e 3 em chat síncrono ${ }^{7}$. Apenas 4 lidaram com ferramentas digitais da chamada web 2.0, sendo em 4 objetos distintos (blogs, Facebook, Orkut e Wikipedia).

Surpreendentemente, 14 artigos não aplicam os indicadores. São exclusivamente metodológicos, não testando seus próprios indicadores. Todavia, assim como é proposto por este artigo, encontramos apenas uma proposta de revisão geral dos indicadores utilizados na área (TRÉNEL, 2004).

Ao todo, foram encontrados 369 códigos para a avaliação da OD (incluindo os idênticos). Excluídas as repetições, ainda há um universo de 229 critérios diferentes aplicados em pesquisas de OD. Isso, inicialmente, apoia o argumento de determinados autores sobre a discrepância nos indicadores utilizados, o que poderia indicar uma dispersão do campo e, em alguma medida, ser uma possível explicação para resultados muito díspares encontrados pelos pesquisadores (DAHLBERG, 2002; JASSEN, KIES, 2005; JENSEN, 2003). Também foi verificado o número de critérios por pesquisa, que variou entre o mínimo de 3 e o máximo de 15, com média de 6,25 e desvio padrão de 2,69. Ou seja, a variação do número de critérios foi, preponderantemente, entre 9 e 5.

A partir do levantamento, agrupamos por aproximação os critérios utilizados pelos analistas da deliberação online, o que resultou em 14 grupos, apresentados na Tabela 1:

\footnotetext{
7 Fóruns de discussão e listas de e-mails são considerados assíncronos pois os participantes não precisam debater simultaneamente. Por outro lado, chats e softwares de conversação (e.g. MSN, Gtalk) são síncronos, pois necessitam que os usuários estejam dialogando no mesmo período temporal.
} 
OPINIÃO PÚBLICA, Campinas, vol. 18, n², novembro, 2012, p. $470-489$

Tabela 1

Relação entre as categorias gerais e o número de critérios

\begin{tabular}{|l|c|}
\hline \multicolumn{1}{|c|}{ CATEGORIA } & QUANT. \\
\hline $\begin{array}{l}\text { I) inclusão formal e simbólica de todos os concernidos ao assunto em } \\
\text { deliberação }\end{array}$ & 25 \\
\hline II) pluralidade e diversidade discursiva e na composição dos debatedores & 32 \\
\hline III) igualdade da dignidade para emitir opinião & 21 \\
\hline $\begin{array}{l}\text { IV) reciprocidade entre os envolvidos na deliberação e a frequência e o tamanho } \\
\text { das falas }\end{array}$ & 72 \\
\hline V) justificação racional, lógica e moral dos argumentos e posições & 83 \\
\hline VI) reflexividade entre os argumentos e posições & 32 \\
\hline $\begin{array}{l}\text { VII) ideal role taking (IRT), entendido como um esforço mental e emocional de } \\
\text { colocar-se na perspectiva do outro (empatia) }\end{array}$ & 8 \\
\hline $\begin{array}{l}\text { VIII) respeito aos argumentos, posições e identidade dos envolvidos na } \\
\text { deliberação, bem como de qualquer outro individuo ou grupo }\end{array}$ & 31 \\
\hline $\begin{array}{l}\text { IX) sinceridade, a necessidade dos participantes serem sinceros em suas } \\
\text { proposições }\end{array}$ & 9 \\
\hline X) as informações disponibilizadas sobre a questão em deliberação & 10 \\
\hline XI) o tópico da discussão & 8 \\
\hline XII) autonomia do poder estatal e econômico & 6 \\
\hline XIII) Common Good Orientation e construtividade das proposições & 16 \\
\hline XIV) outros & 16 \\
\hline
\end{tabular}

Fonte: elaboração dos autores

Em pesquisa similar a esta, Trénel (2004) afirma que são quatro os critérios essenciais para a avaliação de uma deliberação online: Inclusão, Coerência, Racionalidade e Respeito. Contudo, em nossa avaliação, estes acabam sendo demasiadamente extensos, podendo abarcar questões muito amplas e distintas entre si. Categorias menores permitem aplicações mais específicas e mais precisas. Por outro lado, há duas questões a serem ponderadas. Primeiramente, alguns critérios foram pouco adotados, o que indica menor preferência por parte dos pesquisadores. Em segundo lugar, com uma avaliação mais detida dos conceitos de cada grupo de critérios e da respectiva operacionalização, agrupamos algumas categorias, por ocorrer sobreposição conceitual ou na operacionalização. O primeiro grupo foi composto por (V) justificação racional. O segundo por (IV) reciprocidade e (IX) sinceridade. O terceiro grupo composto por (II) pluralidade, (I) inclusão e (XII) autonomia do poder estatal e econômico. O quarto por (VI) reflexividade, (VII) ideal role taking e (XIII) common good orientation. O quinto por (VIII) respeito. O sexto grupo por (III) igualdade da dignidade para emitir opinião. O sétimo, (X) as informações disponibilizadas sobre a questão em deliberação. O oitavo, (XI) o tópico da discussão. E o nono por (XIV) outros. Com isso, adotando os termos mais genéricos, a Tabela 2 apresenta o resultado consolidado. Alguns grupos apresentam números superiores a 59 porque boa parte dos artigos apresentou mais de um critério em cada grupo. 
SAMPAIO, R. C.; BARROS, S. A. R.; MORAIS, R. Como avaliar a deliberação online?...

Tabela 2

Relação entre as categorias agrupadas e o número de critérios

\begin{tabular}{|l|c|}
\hline \multicolumn{1}{|c|}{ CATEGORIA } & QUANT. \\
\hline 1) justificação & 83 \\
\hline 2) reciprocidade & 81 \\
\hline 3) pluralidade & 63 \\
\hline 4) reflexividade & 56 \\
\hline 5) respeito & 31 \\
\hline 6) igualdade & 21 \\
\hline 7) informação & 10 \\
\hline 8) tópico & 8 \\
\hline 9) outro & 16 \\
\hline
\end{tabular}

Fonte: elaboração dos autores

\section{Critérios}

1) JUSTIFICAÇÃO: o grupo compreende a sustentação racional, lógica e moral dos argumentos e posições, numa situação de troca discursiva e crítica recíproca (DAHLBERG, 2004). Neste grupo, juntamos o que alguns autores chamam de argumentação, argumentação racional ou racionalidade. No entanto, independente do termo adotado, a justificação é uma condição necessária para a efetiva racionalização do debate, um dos princípios centrais da legitimidade da esfera pública habermasiana e também da deliberação, algo percebido pelos estudiosos no campo, uma vez que foi o critério mais adotado. Contudo, a condição básica das justificativas é que sejam intelectualmente acessíveis. Segundo Kies (2010), não pode haver deliberação se os cidadãos recorrem à "revelação da autoridade" ou se a justificação é complexa ao ponto de ser acessível apenas para uma minoria intelectual.

Portanto, defende-se que a deliberação não pode se restringir ao discurso racional. Outras formas comunicativas, como a barganha, a retórica e a emoção devem ser consideradas, pois, dificilmente, a argumentação será exclusivamente racional (DRYZEK, 2007). Essa premissa também é considerada em diversos estudos de OD, especialmente na forma de considerar testemunhos, experiências pessoais como formas de justificação (FreELON et al, 2008; MANOSEVITCH, WALKER, 2009).

$\mathrm{Na}$ amostra deste estudo, a justificativa, considerando os diferentes termos empregados, ocorreu 83 vezes, por exemplo, como qualidade do argumento; se são oferecidas razões justificadas para as crenças (FREELON et al, 2008); tamanho do repertório de argumentos (ZHANG, 2007); argumentação para validação externa, validação interna ou alegações (JENSEN, 2003; MIOLA, 2011); formas de justificação (apenas opinião, experiência pessoal explícita, informações de briefing, informações externas, autoridades externas, outros participantes, moderador, não explicitado) (WALES et al, 2010).

2) RECIPROCIDADE: é transversalmente conceituada como o ato de ouvir e responder ao outro; é levar em conta os argumentos apresentados pela alteridade na formulação do próprio ponto de vista, em uma tentativa de construção de consenso (JENSEN, 2003). Segundo Kies (2010), a reciprocidade refere-se ao nível em que a conversação é uma "discussão real". Este critério é uma condição básica para a deliberação, uma vez que se os participantes não ouvem e não consideram uns aos outros não é deliberação, mas apenas monólogo. Sua importância é verificada no estudo, uma vez que se trata do segundo critério mais utilizado pelos estudiosos. Na amostra utilizada, a reciprocidade ocorreu 81 vezes 
OPINIÃO PÚBLICA, Campinas, vol. 18, n², novembro, 2012, p. $470-489$

com diversos modos de operacionalização. Entre outros modos, a medição da reciprocidade é operacionalizada como dialógico ou monológico (JENSEN, 2003; MIOLA, 2011); se inicia um debate, se responde a mensagens prévias (SCHNEIDER, 1997; FREELON et al, 2008; TSALIKI, 2002); se expressa acordo, desacordo, neutralidade ou se interrompe a discussão (TALPIN, MONNOYER-SMITH, 2010).

3) PLURALIDADE: Fazem parte deste grupo os critérios antes categorizados como pluralidade $(\mathrm{N}=32)$, inclusão $(\mathrm{N}=25)$ e autonomia do poder estatal e econômico $(\mathrm{N}=6)$. Estes foram agrupados por entendermos que estas categorias têm como base comum o reconhecimento de que todos os diferentes pontos de vista devem ter oportunidade de se apresentar durante a deliberação para que possam ser avaliados e confrontados. A inclusão diz respeito à preocupação de que todos os concernidos, independente da posição, sejam formalmente incluídos na esfera deliberativa, por exemplo, que tenham as competências e recursos para o uso da internet e que tenham acesso aos ambientes deliberativos (DAHLBERG, 2004; JANSSEN, KIES, 2005; SAMPAIO et al, 2010). Já a pluralidade diz respeito à preocupação direta com a diversidade de opiniões, de perspectivas, nos processos deliberativos, a fim de evitar a concentração em uma determinada opinião e/ou o monopólio de grupos (like-minded) (KIES, 2010; Stromer-Galley, 2007; SzABO, 2009; TSAlikI, 2002). Do mesmo modo, ao se avaliar a autonomia do poder estatal e econômico, busca-se a possibilidade de os indivíduos sustentarem posições contrárias aos interesses destes entes (JANSSEN, KIES, 2004; DAHLBERG, 2001)

Decidiu-se nomear essa categoria como Pluralidade. Além de o indicador ter sido mais utilizado, acreditamos que só haverá diversidade, heterogeneidade de opiniões com a inclusão de diversos grupos. Logo, esta é um pré-requisito daquela. Todos os grupos concernidos, idealmente, devem estar incluídos na deliberação (HABERMAS, 1997), o que, teoricamente, garantiria a pluralidade de visões necessária.

4) REFLEXIVIDADE: é entendida como a consideração da perspectiva alheia ao formular as próprias argumentações e o estabelecimento de posição nas discussões, ou seja, incorporar os argumentos dos outros. No sentido forte de reflexividade, os envolvidos no debate devem reavaliar criticamente os próprios argumentos, posições e valores durante o processo de deliberação (DAHLBERG, 2004).

Por esse motivo, tal indicador ( $\mathrm{N}=32$ ) incorporou o grupo descrito como /deal Role Taking $(\mathrm{N}=8)$. $\mathrm{O}$ IRT é entendido como um esforço mental e emocional de colocar-se na perspectiva do outro. $\mathrm{O}$ desafio é considerar o outro e rever as próprias posições para que o acordo seja construído nos melhores termos o que, no caso, pode ser entendido como a common good orientation ( $\mathrm{N}=16)$ (DAHLBERG, 2004). Logo, entendemos que sejam dois critérios muito similares e não autônomos entre si. Para ser reflexivo, em alguma medida, é necessário (ou, no mínimo, é bastante profícuo) se colocar no lugar do outro. E, ao realizar este esforço, há maior probabilidade de se alcançar a reflexividade.

Nessa categoria, além de reflexividade e IRT, foram agrupados critérios que apareceram como "incorporar os outros argumentos" (WILHELM, 2000; STREET, WRIGHT, 2007), e coerência em escutar outras referências e não utilizar apenas seu ponto de vista para fazer avançar a deliberação (WALES et al, 2010; MendonçA, Pereira, 2010). 
SAMPAIO, R. C.; BARROS, S. A. R.; MORAIS, R. Como avaliar a deliberação online?...

5) RESPEITO: é entendido basicamente como respeito à condição do outro, que é invariavelmente diferente por ser um "não-eu". Em termos práticos, este critério se manifesta no respeito pelos argumentos do outro, respeito a grupos e minorias e manutenção de uma atmosfera de grupo que busca o entendimento comum, apesar das diferenças. Inicialmente, os estudiosos de OD consideravam que qualquer forma de desrespeito presente na discussão inibia completamente a chance de deliberação (DAHLBERG, 2004; WILHELM, 2000). Papacharissi (2004) realizou uma guinada na questão, ao demonstrar que a presença de mensagens rudes não elimina totalmente a possibilidade de outros aspectos deliberativos. Outros estudos demonstram que uma mensagem pode ser rude a um usuário ou argumento e ainda apresentar bons índices nos outros critérios (JENSEN, 2003; STRANDBERG, 2008). Logo, o respeito pode ser avaliado em sua expressão explícita ou implícita (JANSSEN, KIES, 2005); ou também em sua oposição na forma de insultos, mensagens rudes (FrEelon et al, 2008; SAMPAIO et al, 2010). Considerando as medições da presença do respeito e do desrespeito, há 31 ocorrências desse critério.

6) IGUALDADE: por um lado, em uma perspectiva simbólica, este critério diz respeito a que todas as pessoas sejam consideradas igualmente dignas para emitir juízo e opinião sobre o assunto em questão (FREELON, 2010), e, por outro lado, em uma perspectiva prática, se preocupa em evitar desequilíbrios da frequência e do tamanho das falas dos envolvidos na deliberação (SCHNEIDER, 1997). Juntas, estas duas dimensões da igualdade relacionam-se com a pluralidade e a inclusão discursiva, respectivamente, mas a igualdade diz respeito à relação entre indivíduos e não entre ideias. Pelo contrário, as ideias não devem ser iguais, mas confrontar-se na deliberação. Na amostra deste estudo, a igualdade ocorreu 21 vezes, como igualdade discursiva, igualdade do empowerment dos participantes da deliberação, igualdade no direito de apresentar novos argumentos, críticas e/ou questões (MANSBRIDGE et al, 2006; Graham, 2008).

7) INFORMAÇÃO: é entedida como insumo essencial para a construção (e julgamento) de argumentos racionais, lógicos e sustentados na realidade e também é indispensável para uma pluralidade de visões. Assim, mesmo não se tratando diretamente de um indicador deliberativo, os pesquisadores de OD desejam verificar se os participantes buscam ou não informação e quais seriam suas fontes.

Os analistas entendem que as informações podem ser fornecidas pelos promotores da deliberação, mas podem também ser oriundas de conteúdos dos meios de comunicação, estudos técnicos, organismos do Estado ou da experiência direta dos indivíduos. Este critério, com 10 ocorrências em nossa amostra, teve sua medição operacionalizada de modos diversos, por exemplo: a informação é adicional e/ou solicitada (JENSEN, 2003); nível de informação e confiabilidade das afirmativas (TALPIN, MONNOYER-SMITH, 2010); comentários que incluem informações fatuais relacionadas com a questão em debate (MANOSEVITCH, WALKER, 2009); fonte externa (notícias, estudos acadêmicos ou dados governamentais para sustentar uma opinião) (FREELON et al, 2008); e tipo de conteúdo (fato, opinião, questão, declaração) (STRANDBERG, 2008).

8) TÓPICO: assim como a Informação, não é um elemento inerente aos critérios da deliberação, mas é, provavelmente, o único que considera o ambiente online. Aqui há duas interpretações básicas. 
OPINIÃO PÚBLICA, Campinas, vol. 18, n², novembro, 2012, p. $470-489$

Primeiramente, é considerado o tópico do fórum online (thread). Logo, é analisado se as pessoas permanecem nessa discussão ou se elas tendem a abandoná-la antes de alcançarem o entendimento (DAHLBERG, 2004). Em segundo lugar, é considerado o tópico, como assunto/tema da deliberação. Os temas mais polêmicos, com mais divergência e pluralidade de opinião tendem a ser mais deliberativos (KIES, 2010; WILHELM, 2000). Segundo Janssen, Kies (2005), o tópico do debate pode explicar a variação na participação, distribuição de gênero e qualidade da deliberação. Para Stromer-Galley (2007), a discussão do tópico, sem fugas, é importante para que ocorra deliberação. Na amostra de estudos consultados, ocorreram 8 critérios classificados no grupo Tópico, sendo os exemplos mais significativos: discussão focada no tópico inicial, de preferência questões políticas de interesse político (FreELON, 2010); se ideias e informações de fora do debate digital (TSALIKI, 2002).

\section{Discussão e Conclusão}

Consideramos que o objetivo inicial deste artigo, qual seja, desenhar uma cartografia dos critérios empregados na análise de deliberação online, foi alcançado. Há critérios mais utilizados pelos pesquisadores de OD e estes, mesmo com nomes e descrições diferentes, seguem princípios amplos da teoria deliberativa. Assim, este artigo pode facilitar a uniformização dos indicadores de futuras pesquisas, ao mesmo tempo em que abre margem para que o pesquisador aplique um maior ou um menor número de códigos de acordo com seus objetivos.

Como dito, foram encontrados 59 artigos, sendo 50 em língua inglesa. Apesar de admitirmos que a varredura possa não ter alcançado alguns textos (especialmente publicados em livros que não foram indexados), acreditamos que o universo total de artigos não esteja muito distante desse. Isso demonstra que há menos artigos do que nossa expectativa inicial. Por exemplo, em trabalhos similares, Kræmmergaard, Schlichter (2011) encontraram 450 artigos sobre governo eletrônico no período de 2000 a 2009; Sæbø et al (2008) levantaram 131 artigos de e-participação publicados até o ano de 2006 e Medaglia (2011), que deu continuidade à busca por pesquisas de e-participação, encontrou 123 artigos apenas no período de 2006 a 2011, completando 254 pesquisas sobre e-participação. Logo, em nossa avaliação, o número de artigos de deliberação online do corpus pode ser considerado baixo.

Os motivos só poderiam ser apurados corretamente com surveys com os autores, mas é possível oferecer duas hipóteses. Primeiramente, como apontado por Bachtiger et al (2009), diversos investigadores da deliberação online optam pelo uso de surveys, que geram resultados baseados nas opiniões dos indivíduos, e não necessariamente em textos online, que podem não expressar seus verdadeiros pensamentos. Ou ainda, é possível acreditar que uma boa gama dos autores valorizem o survey como um método mais válido de avaliação em relação à análise de conteúdo.

Em segundo lugar, a teoria deliberativa é baseada em valores democráticos, que tendem a ser normativos, e muitas vezes ideais, sendo, consequentemente, intrincados para a aplicação empírica (KIES, 2010). Além disso, há diferentes correntes presentes na teoria deliberativa, que podem valorizar normas ou valores distintos. Häythio (2003), por exemplo, divide os deliberacionistas em dois grupos, os que valorizam os procedimentos (visando maior igualdade na participação) e aqueles que valorizam o resultado legítimo. Além disso, há outras questões importantes ao campo, como por exemplo, autores 
SAMPAIO, R. C.; BARROS, S. A. R.; MORAIS, R. Como avaliar a deliberação online?...

com maior ênfase na racionalidade da deliberação (HABERMAS, 1997; RAWLS, 2002) versus autores que desejam maior espaço para as emoções no processo deliberativo (MANSBRIDGE, 2007); autores com maior inspiração na teoria liberal (GUTMAN, THOMPSON, 2007), maior ênfase na teoria crítica (DRYZEK, 2007) ou mesmo no pluralismo (MANSBRIDGE et al, 2006). Dessa forma, não se poderia falar de uma dispersão ${ }^{8}$ exclusiva da área de deliberação online, mas uma que já advém das diferentes linhas e ênfases da própria teoria deliberativa.

Em terceiro lugar, há que considerar as especificidades de cada objeto. O presente artigo defendeu que há princípios da teoria deliberativa que são especialmente valorizados pelos estudiosos da OD, mas, certamente, as características técnicas do local de aplicação, o tema do debate, a cultura política dos participantes, o empowerment da discussão etc. são fatores que afetam a deliberação seja online seja presencial (JANSSEN, KIES, 2005). O que, certamente, pode se refletir em critérios únicos de certas pesquisas, os quais, além de não representarem incoerência no campo, são vitais para resultados mais acurados.

Por último, percebeu-se que por mais que haja uma gama extensa de critérios utilizados, eles claramente seguem os princípios levantados pelas 8 categorias identificadas nessa pesquisa (Justificação, Reciprocidade, Reflexividade, Respeito, Pluralidade, Igualdade, Informação e Tópico). Apenas se houvesse um número excessivo de categorias ou um montante expressivo de critérios únicos ("outros"), poderia se comprovar o descompasso na área de deliberação online. Em certa medida, o problema está muito mais conectado à taxonomia que a utilizações errôneas ou excessivamente dispersas da teoria deliberativa? .

Além disso, acredita-se que o mapeamento realizado neste artigo, assim como a apresentação dos critérios e de seus diferentes agrupamentos possam ajudar as futuras pesquisas sobre deliberação online. Nesse sentido, os resultados foram apresentados de forma a facilitar que os pesquisadores possam optar por um número menor (categorias mais abrangentes) ou maior (categorias mais específicas) de critérios, de acordo com as necessidades da pesquisa. Os oito indicadores principais aqui apresentados buscam servir como guias das futuras pesquisas da área.

Finalmente, os resultados podem ser úteis para outros estudos em ambientes digitais. Apesar de os princípios da teoria deliberacionista serem muito exigentes para diversos ambientes não políticos (ou não necessariamente democráticos), eles representam princípios importantes para discussões razoáveis, especialmente, quando se trata de tarefas que exigem compromisso, colaboração e respeito entre os participantes, como um jornal participativo, um blog coletivo ou algum wiki.

\footnotetext{
8 Não se defende que a dispersão seja por si negativa. Ela também pode demonstrar que as teorias relacionadas à democracia deliberativas se expandiram ao ponto de apresentar inúmeras entradas, premissas e objetivos, ou seja, também podem estar relacionadas ao grande interesse, à consequente expansão e até à consolidação da área.

${ }^{9}$ Por outro lado, também foi visível que critérios com o mesmo nome eram utilizados com fins bastante distintos entre si. Por exemplo, para Dahlberg (2004), reciprocidade está relacionada ao simples ato de responder a uma mensagem anterior enquanto Jensen (2003) espera que os participantes considerem os argumentos apresentados. Tal definição se assemelha ao critério de reflexividade de Dahlberg. Nesses casos, não foi considerado o nome, mas sim a definição do critério pelo autor.
} 


\section{Referências Bibliográficas}

BACHTIGeR, A.; SHIKANO, S.; PedRINI, S.; RYSER, M. Measuring Deliberation 2.0: Standards, Discourse Types, and Sequenzialization. ECPR General Conference, Potsdam, 2009.

Baek, Y. M; Wojcieszak, M. E.; CarpinI, M. X. D. "Online Versus Face-to-Face Deliberation: Who? Why? What? With What Effects?" New Media \& Society, vol. 13, n 7, p. 135-162, 2011.

Benn, N.; Macintosh, A. Argument Visualization for eParticipation: Towards a Research Agenda and Prototype Tool. International Conference ePart, Delft, 2011 Aug.Sept.

BeSSETTE, J. Deliberative Democracy: The Majority Principle in Republican Government. In: GoldWIN, R. A.; SchAmBRA, W. A. (Orgs.). How Democratic is the Constitution? Washington: AEL Press, 1980.

Black, L.; Burkhalter, S.; Gastil, J.; Stromer-Galley, J. Methods for Analyzing and Measuring Group Deliberation. In: Holbert, L. (Org.). Sourcebook of Political Communication Research: Methods, Measures, and Analytical Techniques. New York: Routledge, 2009.

Bohman, J. Public Deliberation: Pluralism, Complexity and Democracy. Cambridge: MIT Press, 1996.

COHEN, J. Deliberation and democratic legitimacy. In: BOHmAn, J.; WILLIAM, R. (Orgs.). Deliberative Democracy. Essays on Reason and Politics. Cambridge, 1997.

DahlBerg, L. Net-Public Sphere Research: Beyond the 'First Phase'. Javnost-The Public, Liubliana, Eslovênia, vol. 11, n¹, p. $27 \cdot 44,2004$.

DeCIndio, F.; Peraboni, C. Design Issues for Building Deliberative Digital Habitats. Anais do International Conference ePart,Leeds, 2010 June-July.

Delli CarpinI, M. "Gen.com: Youth, Civic Engagement, and the New Information Environment". Political Communication, $\mathrm{n}^{\circ} 17$, p. 341.349, 2000.

Dryzek, J. Theory, Evidence and the Tasks of Deliberation. In: Rosenberg, S.W. (Org.). Deliberation, Participation and Democracy: Can the people govern? New York: Palgrave Macmillan, 2007.

FerReIRA, G. B. "Political debate on weblogs: a virtual public sphere for deliberation?" Communication Studies, vol. 10, p. 213-226, Dec. 2011.

FreELON, D. G. "Analyzing online political discussion using three models of democratic communication". New Media \& Society, Washington, USA, vol. 12, n 7, p. 1172-1190, 2010.

Freelon, D.; Watanabe, M.; Busch, L.; Kawabata, A. Town Halls of the Digital Age: Controversy and Ideology in Online Deliberation (and Beyond). Conference of the Association of Internet Researchers, Copenhagen, Denmark, 2008.

GASTIL, J. "Is face-to-face citizen deliberation a luxury or a necessity?" Political Communication, Filadélfia, PA, vol. 17, p. $357 \cdot 361,2000$.

Gomes, W. Internet e participação política. In: Gomes, W.; MAIA, R. C. M. (orgs.). Comunicação e Democracia: problemas e perspectivas. São Paulo: Paulus, 2008.

GrahAm, T. "Needles in a haystack: a new approach for identifying and assessing political talk in nonpolitical discussion forums". Javnost-The Public, Liubliana, Eslovênia, v. 15, n², p. 17-36, 2008.

Gutmann, A.; Thompson, D. "O que significa democracia deliberativa". Revista Brasileira de Estudos Constitucionais, Belo Horizonte, Ano $1, n^{\circ} 1$, jan./mar. 2007.

Habermas, J. Direito e Democracia: entre facticidade e validade. Rio de Janeiro: Tempo Brasileiro, 1997.

HÄYhtı, T. Can Virtual Spaces Become Political Public Spheres: The Internet Forums of the City of Tampere? Anais do internacional Scanning for the Future, Finland, 2003 June.

JANSSEN, D.; KIES, R. “Online Forums and Deliberative Democracy”. Acta Politica, vol. 40, p. 317-335, 2005.

JENSEN, J. L."Public Spheres on the Internet: Anarchic or Government-Sponsored - A Comparison". Scandinavian Political Studies, vol. $26, n^{\circ} 4,2003$. 
SAMPAIO, R. C.; BARROS, S. A. R.; MORAIS, R. Como avaliar a deliberação online?...

Karamanou, A., Loutas, N., TARABanis, K. ArgVis: Structuring Political Deliberations Using Innovative Visualisation Technologies. International Conference ePart, Delft, 2011 Aug,-Sept.

KIES, R. Promises and Limits of Web-deliberation. New York; Palgrave Macmillan, 2010.

LABCOM. Disponível em: < http://www.labcom.ubi.pt/subp/pagina.php?p=publications\&cod=33>. Acesso em: 15 dez.2011.

LoukIS, E., XenAKIS, A., TSEPERLI, N. Using Argument Visualization to Enhance e-Participation in the Legislation Formation Process. Anais do International Conference e Part, Linz, p. 125-138, 2009 Sept.

ManoseVitch, E.; Walker, D. Reader Comments to Online Opinion Journalism: A Space of Public Deliberation. International Symposium on Online Journalism, 10, Austin, 2009.

Manin, B. "On legitimacy and political deliberation". Political Theory, vol 15, n 3, p. 338-368, Aug. 1987.

Mansbridge, J.; Hartz.KarP, J.; Amengual M.; Gastil, J. "Norms of Deliberation: An Inductive Study". Journal of Public Deliberation, vol. 2, $\mathrm{n}^{\circ} 1,2006$.

MANSBRIDGe, J. "Deliberative democracy" or "democratic deliberation"? In: RosenBERG, S. (org.). Deliberation, Participation and Democracy: Can the People Govern? Basingstoke, UK: Palgrave Macmillan, 2007.

MedagliA, R. eParticipation Research: A Longitudinal Overview. Anais do Proceedings of ePart 2011. Delft, Holanda, p.99.108, 2011.

MendonçA, R.; PereiRa, M. A. Democracia digital e deliberação online: um estudo de caso sobre o VotenaWeb. Congresso Latino Americano de Opinião Pública - Wapor, 4, Belo Horizonte, 2011.

MıLA, E. A Deliberação Online em ambientes institucionais. Um Estudo do Fórum de Discussão do Portal da Câmara dos Deputados. In: MaIA, R. C. M.; Gomes, W.; MARQues, F. P. J. A. (orgs.) .Internet e participação política no Brasil. Porto Alegre: Sulina, 2011.

NOVECK, B. S. "Designing Deliberative Democracy in Cyberspace: The Role of the Cyber-Lawyer". Journal of Science and Technology Law, vol.9, n 1, p. 1.91, 2003.

PAPACHARISSI, Z. "Democracy online: civility, politeness, and the democratic potential of online political discussion groups". New Media and Society, vol. 6, n² 2, p. 259-283, 2004.

KREMMERGAARD, P.; SCHLICHTER, B. R. A comprehensive literature review of the e-government research field over a decade. tGov Workshop 2011 (tGOV11). Brunel University, Londres, 2011 Mars.

RawLS, J. O liberalismo político. São Paulo: Ática, 2000.

Uma teoria de Justiça. São Paulo, Martins Fontes, 2002.

SAEBO, Ø.; RoOSE, J.; FLAK, L. S. "The shape of eParticipation: Characterizing an emerging research area". Government Information Quarterly, vol. 25, n³, p.400-428, 2008.

SAMPAIO, R. C.; MAIA, R. C. M.; MARQUes, F. P. J. A. "Participação e deliberação na internet: um estudo de caso do Orçamento Participativo Digital de Belo Horizonte. Opinião Pública, Campinas, vol. 16, n² 2, p.446.477, nov. 2010.

SAmpaIO, R. C.; BARRos, S. A. R. "Deliberação no jornalismo online: um estudo dos comentários do Folha.com". Intexto, Porto Alegre, vol. 2, n²3 p. 183. 202, Jul./Dez. 2010.

SCHNEIDER, S. M. Expanding the public sphere through computer-mediated communication: Political discussion about abortion in a Usenet newsgroup. PhD Thesis. 189f. 1997. Departament of Political Science da Massachusetts Institute of Technology, 1997.

SILva, F. C. Democracia deliberativa: Avaliando os seus limites. Congresso da Associação Portuguesa de Ciência Política, 2, Lisboa, 2004. Disponível em: <http://www.ces.fe.uc.pt/publicacoes/oficina/203/203.pdf>. Acesso em: 3 jan. 2009

StrandBerg, K. "Public Deliberation goes on-line? An analysis of citizens' political discussions on the internet prior to the finnish parliamentary elections in 2007". Javnost-The Public, vol. 15, n 1, p. 71-90, 2008.

Stromer-Galey, J. “Measuring Deliberation's Content: A Coding Scheme”. Journal of Public Deliberation, vol. 3, 2007. 
OPINIÃO PÚBLICA, Campinas, vol. 18, n², novembro, 2012, p. $470-489$

SzABO, A. Measuring Deliberation Online. Seminar Paper, Universidade de Vaasa, Finlândia, 2009. Disponível em: <http://raatali.files.wordpress.com/2008/09/szabo_seminarpaper_09.pdf> Acesso em: 25 nov. 2009.

TALPIN, J.; Monnoyer-Smith, L. Talking with the wind? Discussion on the quality of deliberation in the Ideal-EU project. IPSA International Conference, Luxemburgo, 2010.

TAMBouris, E.; Dalakiouridou, E.; PANOPOULOU, E.; TARABANIS; K. Evaluation of an Argument Visualisation Platform by Experts and Policy Makers. International Conference ePart, Delft, 2011 Aug. Sept.

TRÉnEL, M. Measuring the quality of online deliberation. Coding scheme 2.4. Berlim, Alemanha: Social Science Research Center Berlin, 2004

TSALIKI, L. "Online Forums and the enlargement of public space: research findings from a European project". Javnost-The Public, v. 9, n’2, p. 95.112, 2002.

Wales, C.; CotteriLl, S.; Smith, G. "Do Citizens 'deliberate' in on-line discussion forums? Preliminary findings from an Internet experiment". Political Studies Association Conference, Edimburgo, 2010.

WARREn, M. E. Institutionalizing Deliberative Democracy. In: RosenberG, S. (Org.). Deliberation, Participation and Democracy: Can the people govern? New York: Palgrave Macmillan, 2007.

WILHELM, A. Democracy in the digital age: challenges to political life in cyberspace. New York: Routledge, 2000.

Wright, S.; Street, J. "Democracy, deliberation and design: the case of online discussion foruns". New Media Society, Londres, vol. 9, p. 849.869, 2007.

ZHANG, W. Diverse and Regulated? A Study on the Relationship Between Online Deliberation and Structural Design. Annual Meeting of the International Communication Association, São Francisco, 2007. Disponível em: <http://www.allacademic.com/meta/p169136_index.html>. Acesso em: 29 dez. 2010.

\section{ANEXO 1: CORPUS para verificação dos critérios}

1. Bachtiger, A.; Shikano, S.; Pedrini, S.; Ryser, M. Measuring Deliberation 2.0: Standards, Discourse Types, and Sequenzialization. ECPR General Conference, Potsdam, 2009. Disponível em: <http://ash.harvard.edu/extension/ash/docs/baechtiger.pdf>. Acesso em: 13 fev. 2011.

2. BeIERLE, T. An Evaluation of the National Dialogue on Public Involvement in EPA Decisions. Washington, D. C.: Resources for the Future, 2002. Disponível em: <http://www.rff.org/rff/Documents/RFF.RPT-demonline.pdf>. Acesso em: 28 mar. 2011.

3. Coleman, S.; Blumler, J. G. E.Democracy from Above. In: Coleman, S.; Blumler, J. G. (orgs.). The internet and democratic citizenship: theory, practice and policy. Cambridge, UK: Cambridge University Press, 2009.

4. DAHLBERG, L. "The Internet and Democratic Discourse: Exploring The Prospects of Online Deliberative Forums Extending the Public Sphere". Information, Communication \& Society, v. 4, n. 4, p. 615.633, 2001.

5. FREELON, D. G. "Analyzing online political discussion using three models of democratic communication". New Media \& Society, Washington, USA, v. 12, n. 7, p. 1172-1190, 2010.

6. Freelon, D.; Watanabe, M.; Busch, L.; KawabatA, A. Town Halls of the Digital Age: Controversy and Ideology in Online Deliberation (and Beyond). Conference of the Association of Internet Researchers, Copenhagen, Denmark, 2008.

7. Graham, T. "Needles in a haystack: a new approach for identifying and assessing political talk in nonpolitical discussion foruns". Javnost-The Public, Liubliana, Eslovênia, v. 15, n. 2, p. 17.36, 2008.

8. What's Wife Swap Have to Do with It? Talking Politics Online. International Conference on Online Deliberation, 4, Leeds, UK, 2010. Disponível em:

9. <http://www.od2010.dico.unimi.it/docs/proceedings/Proceedings_OD2010.pdf>. Acesso em: 28 mar. 2011.

10. Hagemann, C. "Participation in and contends of two Dutch political party discussion lists on the internet". Javnost-The Public, Liubliana, Eslovênia, v. 9, n. 2, p. 61-76, 2002. 
SAMPAIO, R. C.; BARROS, S. A. R.; MORAIS, R. Como avaliar a deliberação online?...

11. Janssen, D.; KIES, R. “Online Forums and Deliberative Democracy”. Acta Politica, v. 40, p. 317.335, 2005.

12. JENSEN, J. L. "Public Spheres on the Internet: Anarchic or Government-Sponsored - A Comparison". Scandinavian Political Studies, v. 26, n. 4, 2003.

13. JANKOWSKI, N.; VAN OS, R. Internet-based political discourse: A case study of electronic democracy in the city of Hoogeveen. In: SHANE, P. M. (orgs.). Democracy Online: the prospects for political renewal through the Internet. New York: Routledge, 2004.

14. KARLSSON, M. What does it take to make online deliberation happen? - A comparative analysis of 28 online discussion forums. International Conference on Online Deliberation, 4, Leeds, UK, 2010. Disponível em: <http://www.od2010.dico.unimi.it/docs/proceedings/Proceedings_OD2010.pdf>. Acesso em: 27 mar. 2011.

15. KIES, R. Promises and Limits of Web-deliberation. New York: Palgrave Macmillan, 2010.

16. KIES, R.; WOJCIK, S. European Web-Deliberation: Lessons From the European Citizens Consultation. International Conference on Online Deliberation, 4, Leeds, UK, 2010. Disponível em: <http://www.od2010.dico.unimi.it/docs/proceedings/Proceedings_OD2010.pdf>. Acesso em: 28 mar. 2011.

17. LOWRY, M. B. "Online public deliberation for a regional transportation improvement decision". Transportation, v. 37 , n. 1 , p. $39 \cdot 58,2009$.

18. Monnoyer-Smith, L.; TALPIN, J. Participatory Frames in Deliberative Devices: the Ideal-EU Case Study. International Conference on Online Deliberation, 4, Leeds, UK, 2010. Disponível em: <http://www.od2010.dico.unimi.it/docs/proceedings/Proceedings_OD2010.pdf>. Acesso em: 28 mar. 2011.

19. Manosevitch, E.; Walker, D. Reader Comments to Online Opinion Journalism: A Space of Public Deliberation. International Symposium on Online Journalism, 10, 2009, Austin. Disponível em: <http://online.journalism.utexas.edu/2009/papers/ManosevitchWalker09.pdf>. Acesso em: 20 jan. 2010.

20. MARQUES, A. A deliberação online como uma das dimensões políticas da comunicação mediada por computador: reflexões teórico-metodológicas. Congresso Brasileiro de Ciências da Comunicação -Intercom, 33, Caxias do Sul, RS. 2010. Disponível em: <http://www.intercom.org.br/papers/nacionais/2010/resumos/R5-3056-1.pdf>. Acesso em: 29 dez. 2010.

21. MendonçA, R.; Pereira, M. A. Democracia digital e deliberação online: um estudo de caso sobre o VotenaWeb. Congresso Latino Americano de Opinião Pública - Wapor, 4, Belo Horizonte, BH, 2011.

22. MIOLA, E. "A deliberação online em ambientes institucionais. Um estudo do fórum de discussão do portal da Câmara dos Deputados". Contemporânea, Salvador, vol. 7, n. 2, p. 1·24, 2009.

23. MISNIKOV, Y. Discursive qualities of public discussion on the Russian Internet: Testing the Habermasian communicative action empirically. International Conference on Online Deliberation, 4, Leeds, UK, 2010. Disponível em: <http://www.od2010.dico.unimi.it/docs/proceedings/Proceedings_OD2010.pdf>. Acesso em: 28 mar. 2011.

24. RHeE W. J.; KIM, E. The Effect of Online Deliberation on Political Discussion Efficacy: A Field Experiment on the Internet Discussion Groups. International Communication Association, São Francisco, 2007. Disponível em: <http://www.allacademic.com//meta/p_mla_apa_research_citation/0/9/2/3/0/pages92309/p92309.2.php> Acesso em: 13 fev. 2011

25. SAmpaIO, R. C.; Barros, S. A. R. "Deliberação no jornalismo online: um estudo dos comentários do Folha.com". Intexto, Porto Alegre, v. 2, n. 23 p. 183. 202, jul./dez. 2010.

26. SAMPaIO, R. C.; DANTAS, M. "Deliberação Online em Fóruns de Discussão: Um estudo dos potenciais democráticos do Cidadão Repórter". Contracampo, Niterói, n’. 22, p. 159-177, fev. 2011.

27. Sampaio, R. C.; MalA, R. C. M.; Marques, F. P. J. A. "Participação e deliberação na internet: Um estudo de caso do Orçamento Participativo Digital de Belo Horizonte". Opinião Pública, Campinas, v. 16, n. 2, p.446.477, nov. 2010

28. SCHNEIDER, S. M. Expanding the public sphere through computer-mediated communication: Political discussion about abortion in a Usenet newsgroup. Tese de Doutorado. 189f. 1997. Departamento de Political Science da Massachusetts Institute of Technology, 1997.

29. Smith, G.; Wales, C.; John, P.; CotTerill, S.; Sturgis, P.; Stoker, G.; NAmURA, H. Measuring the 'deliberative quality' of an online experimental mini-public: methodology and early results. ECPR General Conference, Potsdam, Alemanha, 
2009. Disponível em:

<http://www.civicbehaviour.org.uk/documents/Measuringthedeliberativequalityofanonlineexperimentalmini. publicECPR_000.pdf>. Acesso em: 28 mar. 2011.

30. StrandBerg, K. "Public Deliberation goes on-line? An analysis of citizens' political discussions on the internet prior to the finnish parliamentary elections in 2007". Javnost-The Public, v. 15, n. 1, p. 71-90, 2008.

31. Stromer-Galey, J. "Measuring Deliberation's Content: A Coding Scheme". Journal of Public Deliberation, v. 3, 2007

32. SzABO, A. Measuring Deliberation Online. Seminar Paper, Uni of Vaasa, Filand, 2009. Disponível em: <http://raatali.files.wordpress.com/2008/09/szabo_seminarpaper_09.pdf> Acesso em: 25 nov. 2009.

33. PAPACHARISSI, Z. "Democracy online: civility, politeness, and the democratic potential of online political discussion groups". New Media and Society, v. 6, n.2, p. 259.283, 2004.

34. TALPIN, J.; Monnoyer-Smith, L. Talking with the wind? Discussion on the quality of deliberation in the Ideal-EU project. IPSA International Conference, Luxemburgo, 2010.

35. TRÉNEL, M. Facilitating deliberation online: What difference does it make? Draft paper submitted to the $2 n d$ Annual Conference on Online Deliberation. Annual Conference on Online Deliberation, 2, Stanford, CA, 2005. Disponível em: <http://www.online-deliberation. net/conf2005/viewabstract. php?id=51>. Acesso em: 13 fev. 2011.

36. TSALIKI, L. "Online Forums and the enlargement of public space: research findings from a European project". Javnost-The Public, v. 9, n. 2, p. 95-112, 2002.

37. Wales, C.; CotTerilL, S.; SMith, G. Do Citizens 'deliberate' in on-line discussion forums? Preliminary findings from an Internet experiment. Political Studies Association Conference, 2010, Edinburgh. Disponível em: <www.psa.ac.uk/journals/pdf/5/2010/904_585.pdf>. Acesso em: 23 out. 2010.

38. WiLheLM, A. "Democracy in the digital age: challenges to political life in cyberspace". New York: Routledge, 2000.

39. WRIGHT, S.; STREET, J. "Democracy, deliberation and design: the case of online discussion foruns. New Media Society, Londres, v. 9, p. 849.869, 2007.

40.

Wu M. "Measuring Political Debate On The Chinese Internet Forum". Javnost-The Public, v. 15, n.2, p. 93-110 2008.

41. ZHANG, W. Are online discussions deliberate? A case study of a Chinese online discussion board. International Conference Communication and Reality, 3, Barcelona, 2005. Disponível em: <http://cicr.blanquerna.url.edu/2005/Abstracts/PDFsComunicacions/vol1/02/ZHANG_Weiyu.pdf>. Acesso em: 28 mar. 2011

42. ZHANG, W. Diverse and Regulated? A Study on the Relationship Between Online Deliberation and Structural Design. Annual Meeting of the International Communication Association, 2007, São Francisco. Disponível em: <http://www.allacademic.com/meta/p169136_index.html>. Acesso em: 29 dez. 2010.

43. TRÉnEL, M. Measuring the quality of online deliberation. Coding scheme 2.2. Unpublished paper. Social Science Research Center Berlin, 2004, Germany. Disponível em: <http://www.wz-berlin.de/ trenel/tools/qod_2_0.pdf>. Acesso em: 8 dez. 2011.

44. FerReIRA, G. "Political debate on weblogs: a virtual public sphere for deliberation?" Estudos em Comunicação, Covilhã, n 10, p. 213-226, 2011. Disponível em: <http://www.ec.ubi.pt/ec/10/pdf/EC10-2011Dez-12.pdf>. Acesso em: 8 dez. 2011.

45. Stromer-Galey, J. Decoding Deliberation Online. Second Conference on Online Deliberation, 2005, Palo Alto, California. Disponível em: <http://www.online-deliberation.net/conf2005/viewpaper.php?id=22>. Acesso em: 8 dez. 2011.

46. LISTON, V.; HARRIS, C.; O'TOOLE, M. Computer-mediated deliberation for citizen self-government: A theoretical design of an integrated policy process. Annual Conference Political Studies Association of Ireland, 2011. Disponível em:<http://sowit.eu/documents/Computer_mediated_deliberation_graphs.pdf>. Acesso em: 8 dez. 2011. 
SAMPAIO, R. C.; BARROS, S. A. R.; MORAIS, R. Como avaliar a deliberação online?...

47. ALBRECHT, S. Political Discourses as Communicative Networks: An Investigation of Distortions in Online Deliberation. Annual Meeting of the International Communication Association, Dresden, Germany, 2006. Disponível em: <http://www.allacademic.com/meta/p93151_index.html>. Acesso em: 8 dez. 2011.

48. WANG, K. Democratic Deliberation in Online Consultation Forums: A Study of the Casino Debate in Singapore. In: Shedletsky, L .\& Aitken, J. (Orgs.). Cases on Online Discussion and Interaction: Experiences and Outcomes, IGI Global, p. 263.281, 2010. Disponível em: <www.irma-international.org/chapter/democratic-deliberation-onlineconsultation forums/43669/>. Acesso em: 8 dez. 2011.

49. Robertson, S.; Vatrapu, R.; Medina, R. "Off the wall political discourse: Facebook use in the 2008 U.S. presidential election". Information Polity, v. 5, n’.1, 2010. Disponível em: <https://iospress.metapress.com/content/gl325w55p43gt850/resourcesecured/?target=fulltext.pdf>. Acesso em: 8 dez. 2010.

50. JANSSEN, D.; KIES, R. Online Forums and Deliberative Democracy: Hypotheses, Variables and Methodologies. Conference on Empirical Approaches to Deliberative Politics, Florence, 2004. Disponível em: $<$ http://edc.unige.ch/edcadmin/images/onlineforums.pdf>. Acesso em: 8 dez. 2011.

51. Monnoyer-Smith, L.; WoJcIK, S. Technology and the Quality of Public Deliberation. A Comparison Between On and Off-line Participation. 61st Conference of the International Communication Association, 2011. Disponível em:<http://hal.archivesouvertes.fr/docs/00/59/82/73/PDF/Proposal_final.pdf>Acesso em: 8 dez. 2011.

52. ALVES, N. A conversação cívica sobre a questão do aborto em redes sociais na internet. Dissertação de Mestrado em Comunicação Social do Programa de PósGraduação em Comunicação, Faculdade Cásper Líbero, São Paulo, 2011. Disponível em: <http://www.casperlibero.edu.br/rep_arquivos/2011/04/06/1302108566.pdf>. Acesso em: 8 dez. 2011.

53. IASULAITIS, S. As possibilidades da ciberdemocracia: as modalidades de participação política em websites eleitorais. Anais do XI Conlab (Congresso Luso Afro Brasileiro de Ciências Sociais), Salvador, 2011. Online Deliberation Among Regional Civil Society Groups - The Case of the Caribbean. Tese de Doutorado, Faculdade de Políticas Públicas, Geórgia Institute of Technology, Geórgia, 2010.

54. MALVA, R. Deliberação Pública Online: Análise da construção dos processos deliberativos na esfera pública. Anais do $6^{\circ}$ Interprogramas de Mestrado da Faculdade Cásper Líbero, São Paulo, 2010. Disponível em: <http://www.casperlibero.edu.br/rep_arquivos/2011/04/01/1301679065.pdf>. Acesso em: 8 dez. 2011.

55. GRAHAM, T. The use of expressives in online political talk: impeding or facilitating the normative goals of deliberation? Proceedings of the 2nd IFIP WG 8.5 international conference on Electronic participation, 2010. Disponível em: <https://springerlink3.metapress.com/content/j2722447ph64223j/resource-

secured/?target=fulltext.pdf\&sid=5k2px3ystapy4jlof1cdvmno\&sh=www.springerlink.com>. Disponível em: 20 dez. 2012.

56. DUNNE, K. Cross Cutting Discussion: A form of online discussion discovered within local political online forums. Information Polity 14, p. 219-232, 2009. Disponível em:

<http://cnbrewer.iweb.bsu.edu/cross\%20cutting.pdf>. Acesso em: 8 dez. 2011.

57. BLACK, L; WELSER, H.; COSLEY, D.; DeGroot, J. "Self-Governance Through Group Discussion in Wikipedia: Measuring Deliberation in Online Groups". Small Group Research, v.42, n’.5, p.595-634. 2011.

58. KoOP, R.; JANSEN, H. "Political Blogs and Blogrolls in Canada Forums for Democratic Deliberation?" Social Science Computer Review, 27, 2009. Disponível em: <http://ssc.sagepub.com/cgi/content/abstract/27/2/155>. Acesso em: 8 dez. 2011.

59. Bentivegna, S. Talking Politics on the net. Research Paper Press Politics Harvard University, 1998. Disponível em: <http://citeseerx.ist.psu.edu/viewdoc/download?doi=10.1.1.173.2361\&rep=rep1\&type=pdf>. Acesso em: 20 dez. 2011.

Rafael Cardoso Sampaio ·cardososampaio@yahoo.com.br

Samuel Anderson Rocha Barros - samuel.barros77@gmail.com

Ricardo Morais·rm.ricardomorais@gmail.com

Submetido à publicação em outubro de 2011. Versão final aprovada em dezembro de 2011. 